\title{
Articles
}

The Person and the Challenges

Volume 1 (2011) Number 1, p. 13-23

Józef Stala

The Pontifical University of John Paul II in Cracow, Poland

\section{Die Person und die Herausforderungen der Gegenwart im Licht der Nachfolge und der Lehre des Heiligen Vaters Johannes Pauls II. "The Person and the Challenges" - ein internationales wissenschaftliches Periodikum}

\section{The Person and the Modern Challenges in the Light of the Ministry and Teaching of Pope John Paul II. "The Person and the Challenges" - the International Scientific Journal}

\begin{abstract}
The teachings of Holy Father John Paul II constantly remind us of the importance of human dignity. The anthropological focus of John Paul II is an essential element of his thoughts. The human being in the image of God, has a unique, inherent value. It was always God's plan that men, despite being creatures, would freely participate in God's inner life - in Love. The dignity and value of humanity is established in creation, but is fully realised and expressed in the redemption of Jesus Christ. Therefore, the author of this article presents the person who faces modern challenges, but in the light of the ministry and teaching of Pope John Paul II. This is a direct introduction to the new international scientific journal "The Person and the Challenges: The Journal of Theology, Education, Canon Law and Social Studies Inspired by Pope John Paul II". This journal is being published at the Theology Faculty, Section in Tarnów of the Pontifical
\end{abstract}




\section{The Person and the Challenges \\ 14

University of John Paul II in Cracow since 2011 - the year of the beatification of the Holy Father John Paul II, which also implies and guarantees, that it will become a platform for exchanging ideas, evaluations, reports and studies on the person and contemporary challenges in the light or having regard to the teaching of John Paul II.

\section{Keywords}

The person, the human being, challenges, Pope John Paul II, scientific journal „The Person and the Challenges”.

Den Bezugspunkt für das wissenschaftliche Periodikum „The Person and the Challenges: The Journal of Theology, Education, Canon Law and Social Studies Inspired by Pope John Paul II" bilden vor allem die Gedanken, Reflexionen und die Lehre Papst Johannes Pauls II., der den Wert der Person hoch einschätzte und deshalb immer wieder ihre Würde, ihre Rechte und ihre Pflichten unterstrich. Während seines Pontifikats in den Jahren von 1978 bis 2005 bereicherte Johannes Paul II. die Lehre der Kirche durch eine überwältigende Anzahl unterschiedlicher Dokumente: Enzykliken, Adhortationen, Bullen, Apostolische Konstitutionen, Briefe, Botschaften, Katechesen und Ansprachen. Gleichzeitig lässt sich im Rückblick seine ganz spezielle Art erkennen, wie er mit seinen Zuhörern in Kontakt getreten ist, insbesondere mit den Jugendlichen, die für sein gesprochenes Wort weitaus offener waren als für das geschriebene. Daher erscheint es unerlässlich, gerade zu Beginn des einundzwanzigsten Jahrhunderts, eines Jahrhunderts, das von großen Fortschritten in der Zivilisation, von technischem Denken und der Entwicklung der Demokratie, aber auch von Bedrohungen für die integrale Entwicklung des Menschen gezeichnet ist, an die Ausführungen anzuknüpfen, die Johannes Paul II. hinterlassen hat. In dem vorliegenden Artikel werden sowohl die Person als auch die vor ihr liegenden Herausforderungen der Gegenwart im Licht der Nachfolge und der Lehre des Heiligen Vaters Johannes Pauls II. vorgestellt. Damit soll auch das neue, internationale wissenschaftliche Periodikum präsentiert und auf sein Erscheinen verwiesen werden.

\section{Der Mensch - die Person als Bezugspunkt}

Die gewaltigen zivilisatorischen, politischen, ökonomischen, gesellschaftlichen, kulturellen und religiösen Umbrüche beeinflussen die Ausgestaltung neuer Lebensmodelle für den Menschen des einundzwanzigsten Jahrhunderts. Es 
verändern sich die Wertestrukturen im sozialen und im individuellen Leben, in Folge dessen modifizieren sie die geplanten und realisierten Ziele wie auch die Art und Weise des Handelns. Erziehung und Bildung in den Schulen jeglichen Niveaus zielen ganz deutlich überwiegend darauf ab, Wissen, Fertigkeiten und Können zu vermitteln, damit ein entsprechender Arbeitsplatz gefunden und die berufliche Karriere gestartet werden kann. In der modernen Bildungswirklichkeit existieren viele Systeme und Modelle für die Erziehung, von denen lediglich wenige mit dem christlichen Denken korrespondieren, während die Mehrzahl sich auf völlig abweichende Fundamente beruft. Der grundlegende Unterschied liegt vor allem in den philosophischen anthropologischen Grundsätzen und den daraus folgenden weiteren Konsequenzen. Denn das Erziehungssystem trägt eine - manchmal deutliche, nicht selten verborgene - Vision vom Menschen und der Wirklichkeit in sich, die als logische Konsequenz der geäußerten Thesen gewichtige Konzeptionen und Implikationen mit sich bringt.

Die Zivilisation, welche die moderne Gesellschaft formt, setzt vor allem auf Fortschritt, Wissenschaft, Technik und Produktion, daher bezieht sie sich auf den Menschen nicht unmittelbar als Person sondern auf die Vielzahl und die Verschiedenheit der Produkte, die der Mensch lediglich benutzt - konsumiert. Darum besteht, trotz des hohen Niveaus der technisierten Zivilisation, eine beunruhigende Erscheinung der modernen Wissenschaft in der Abwendung von ihrem eigentlichen Ziel, der Humanisierung der Welt ${ }^{1}$. Aktuelle Zivilisationsprogramme, wie auch die derzeit großen Veränderungen unterliegende Kultur, bedienen sich der Schwäche des Menschen, indem sie ihn zu Hilf- und Wehrlosigkeit degradieren, ihn seiner Geistlichkeit und damit seiner Identität berauben ${ }^{2}$. Dagegen ist die Person in ihrer Individualität niemals nur ein Teil, ein Produkt oder eine Nummer. Die Person ist immer ein Wesen, das in der Unterscheidung von ,jemand anderem” existiert. Daher stellen alle Taten und Erzeugnisse des Menschen, die es in den Zivilisationen und Kulturen gibt, lediglich eine Welt der Mittel dar, derer sich der Mensch bedient, um an sein eigentliches Ziel zu gelangen ${ }^{3}$.

Eines der größten Probleme der modernen Zivilisation stellt eine unrichtige Anthropologie dar, die sich vor allem darum bemüht, den Menschen seiner geistlichen Sphäre zu berauben. Indem sie den Menschen gerade falsch definiert, steht sie für alle Handlungen, die ihn auf instrumentelle Weise behandeln, weil

${ }^{1}$ Vgl. Evangelium vitae 22-23; Redemptor hominis 16.

${ }^{2} \mathrm{Vgl}$. Gratissimum sane 13.

${ }^{3}$ Vgl. Christifideles laici 37; Familiaris consortio 43; Evangelium vitae 13, 75. 
sie seine Würde gering achten ${ }^{4}$. Darüber hinaus präsentieren Philosophien und Ideologien des zwanzigsten und einundzwanzigsten Jahrhunderts bei der Vorstellung des Menschen als Subjekt eine derartige Vision vom Menschen, die es ihm ermöglicht, an die Stelle Gottes zu treten. Bei dieser Auffassung werden Gott und der Mensch zu Konkurrenten um die Wirklichkeiten, zwischen denen man eine Wahl treffen muss: entweder Gott oder der Mensch. Wenn der Mensch als höchster Wert anerkannt wird, dann verliert ein derartiger Humanismus auch die Vision vom Menschen als Individuum und Person. Von daher besteht der große Bedarf, sich deutlich für einen Humanismus einzusetzen, der es dem Menschen erlaubt, vollständig und integral auf seine Natur als Person zu blicken und dabei ihre transzendente Dimension zu berücksichtigen.

Die Würde der Person ist das unverletzbare Eigentum jedes Menschen, weil sie sich auf die Individualität und die Einzigartigkeit jeder Person beruft. Der Mensch ist das einzige Wesen, das sich letztendlich als Person erfüllt, das sich selbst gehört, sich selbst beherrscht, das für sich und durch sich selbst antwortet. Er ist das Zentrum und die Spitze all dessen, was auf der Erde existiert: kein anderes sichtbares Wesen hat eine ihm vergleichbare Würde.

Dennoch ist der Mensch, der dank seiner persönlichen Subjektivität mit einer großen Personenwürde beschenkt ist, auch ein Wesen des Zufalls. Deshalb findet der Mensch sich selbst, sein eigenes „Ich” und das „Ich” seines Mitmenschen letztendlich erst dann, wenn er sich selbst als Geschenk seines Persönlichen Spenders betrachten kann. Denn die Struktur des Menschen als Person besitzt oberste Priorität und ist gleichzeitig eine Aufforderung, am Heilsplan Gottes mitzuwirken. Durch die Berufung zur Existenz als konkreter Mensch, hat Gott ihn auch mit den Möglichkeiten ausgestattet, in seiner Entwicklung zu einem Ebenbild Seiner Person zu werden. Dank dessen ist jeder Mensch nicht nur das Bild seiner Eltern sondern ebenso ein Zeichen Gottes und Sein Spiegelbild. Der Wert der Person ist weder verdient noch bedingt, das heißt er hängt weder von ihrem Gesundheitszustand, von ihrem Bewusstseinsstand, von ihrem Entwicklungsgrad noch von ihren moralischen Verdiensten $\mathrm{ab}^{5}$. Jeder Mensch ist aus seiner Natur heraus zur unablässigen Weiterentwicklung berufen, was ihn natürlicherweise immer zu Gott als seiner Quelle hinführt. Das Streben nach Heiligkeit durch die Ausrichtung auf Gott stellt daher die höchste Ebene des menschlichen Daseins dar. Indem der Mensch sein letztendliches Ziel realisiert, bestätigt er sich folglich

\footnotetext{
${ }^{4}$ Vgl. Evangelium vitae 22-23; Christifideles laici 4-5; Centesimus annus 13.

${ }^{5}$ Vgl. Gratissimum sane 16.
} 
am besten und verwirklicht sich am tiefgründigsten. Denn kein Geschöpf ist in der Lage, das Verlangen des Menschen nach Glück zu befriedigen ${ }^{6}$.

Allein die Liebe, die tiefste Quelle der geistlichen Entwicklung des Menschen, vermag der Grund dafür zu sein, dass in den zwischenmenschlichen Beziehungen schließlich das erwartete gegenseitige Verständnis und die Achtung fremder Ansichten und Vorlieben vorherrschen. Im Laufe seiner Entwicklung wird sich der Mensch bewusst, dass der letztendliche Sinn seiner körperlichen Existenz, der physischen und der geistlichen Sphäre, darin besteht, in Kontakt zu treten, die Begegnung mit sich selbst, dem Mitmenschen und Gott zu suchen. Daher erlebt sich der Mensch nicht in seinen einzelnen Elementen sondern als Gesamtheit, als ganzheitliches Selbst, als Person. Der Personalismus unterstreicht die Tatsache als einen der wesentlichen Bereiche der Person, dass der Mensch über die Fähigkeit verfügt, sich mit anderen Personen zu treffen und auszutauschen, dass die Qualität seiner zwischenmenschlichen Bindungen über die Qualität seines Lebens sowie über die Art entscheidet, wie er mit seiner Freiheit umgeht. Der Mensch tritt mit seinem Mitmenschen sowohl in der menschlichen Gemeinschaft in Beziehung als auch dadurch, dass er sich Jesus Christus gegenüber öffnet, wodurch er erst entdecken kann, wer er wirklich ist und wie er seine eigene Berufung realisieren kann. Gerade im Kontext der postmodernen Zivilisation ist eine Bildung auf die persönliche und religiöse Reife des Menschen hin aufzunehmen. Ein auf dem Personalismus basierendes Bildungsprogramm muss den ganzen Menschen berücksichtigen: Es muss ihn dabei unterstützen seine Körperlichkeit, seine Physis und seine Geistlichkeit zu verstehen, damit er durch das Entdecken seiner Identität mit sich selbst, mit seinem Mitmenschen und mit Gott auf kluge und verantwortliche Weise in Beziehung treten kann. Im derzeitigen kulturellgesellschaftlichen Kontext geht des vor allem um die Entwicklung einer von Liebe erfüllten Hinwendung an den Mitmenschen sowie um das Engagement auf den unterschiedlichen Ebenen des Gesellschaftslebens, unter anderem in Kinderund Jugendorganisationen, im Freiwilligendienst und in karitativen Aktionen.

\section{2. „The Person and the Challenges: The Journal of Theology, Education, Canon Law and Social Studies Inspired by Pope John Paul II"}

Es ist zu bemerken, dass die Errungenschaften der Wissenschaftler aus Polen in den Bereichen der humanistischen und sozialen Wissenschaften,

${ }^{6}$ Vgl. Gaudium et spes 19-21; Dominum et vivificantem 56; Pastores dabo vobis 7; Christifideles laici 34. 
der Philosophie und der Theologie weltweit noch wenig bekannt sind, was insbesondere den Einschränkungen im Hinblick auf die polnische Sprache geschuldet ist. Dies ist unter anderem der Grund dafür, an der Theologischen Fakultät, Sektion Tarnów, der Päpstlichen Universität Johannes Paul II. in Krakau das internationale, halbjährlich erscheinende Wissenschaftsperiodikum „The Person and the Challenges: The Journal of Theology, Education, Canon Law and Social Studies Inspired by Pope John Paul II" herauszugeben. Die vorliegende Initiative schreibt sich hervorragend in die Seligsprechung der Heiligen Vaters Johannes Pauls II. ein, die am 1. Mai 2011 im Vatikan vollzogen wurde ${ }^{7}$, und bezieht sich auf das Wirken der Theologischen Fakultät, Sektion Tarnów, und der gesamten Päpstlichen Universität Johannes Paul II. in Krakau, deren Ziel darin besteht, das Vermächtnis des Glaubens zu vertiefen und Forschungen im Bereich der Philosophie und anderer wissenschaftlicher Disziplinen durchzuführen ${ }^{8}$. „Die Päpstliche Universität Johannes Paul II. in Krakau ist eine kirchliche Universität, kanonisch errichtet durch den Heiligen Stuhl. Gemäß der Bedürfnisse der Kirche in Polen führt sie die historische Mission der Theologischen Fakultät der Jagiellonen-Universität und der Päpstlichen Theologischen Akademie in Krakau fort. Die Päpstliche Universität Johannes Paul II. in Krakau ist eine kirchliche Hochschule, deren Identität und Wirken durch die Apostolische Konstitution Sapientia christiana bestimmt ist. Die Universität nimmt die Forschung und die Weitergabe theologischen Wissens und anderer humanistischen

\footnotetext{
${ }^{7}$ „Vor nunmehr sechs Jahren befanden wir uns auf diesem Platz zur Begräbnisfeier von Papst Johannes Paul II. Groß war der Schmerz über den Verlust, aber noch größer war die Erfahrung einer unendlichen Gnade, die Rom und die ganze Welt umfing: die Gnade, die wie die Frucht des ganzen Lebens meines geliebten Vorgängers und besonders seines Zeugnisses im Leiden war. Schon an jenem Tag spürten wir den Duft seiner Heiligkeit ausströmen, und das Volk Gottes hat auf viele Weisen seine Verehrung für ihn zum Ausdruck gebracht. Daher wollte ich, dass sein Seligsprechungsprozess unter entsprechender Beachtung der Vorschriften der Kirche ziemlich rasch vorangehen konnte. Und heute ist der erwartete Tag gekommen; er ist schnell gekommen, weil es dem Herrn so gefallen hat: Johannes Paul II ist selig... Heute erstrahlt vor unseren Augen im vollen geistlichen Licht des auferstandenen Christus die Gestalt des geliebten und verehrten Johannes Paul II. Heute wird sein Name der Schar der Heiligen und Seligen hinzugefügt, die er während der fast 27 Jahre seines Pontifikates heilig- und seliggesprochen hatte. Dabei hatte er nachdrücklich an die allgemeine Berufung zum hohen Maß des christlichen Lebens - zur Heiligkeit - erinnert, wie sie die Konzilskonstitution Lumen gentium über die Kirche bekräftigt hatte. Alle Glieder des Volkes Gottes - Bischöfe, Priester, Diakone, Laien, gottgeweihte Männer und Frauen - wir alle sind auf dem Weg zur himmlischen Heimat, in welche uns die Jungfrau Maria vorausgegangen ist, die mit dem Geheimnis Christi und der Kirche auf einzigartige und vollkommene Weise verbunden ist." Predigt Benedikts XVI. anlässlich der Seligsprechung Johannes Pauls II., Vatikan, 1. Mai 2011, http:// www.vatican.va/holy_father/benedict_xvi/homilies/2011/documents/hf_ben-xvi_hom_20110501 beatificazione-gpii_ge.html (19.08.2011).

${ }^{8}$ Vgl. Statut der Päpstlichen Universität Johannes Paul II. in Krakau, Nr. 5.
} 
Wissenschaften auf, verleiht berufliche Titel, akademische Grade und den Titel eines Wissenschaftlers mit Zustimmung des Heiligen Stuhls"9. Dagegen setzt die „Theologische Fakultät, Sektion Tarnów, die Arbeit des Theologischen Instituts in Tarnów fort. Das Spezifikum der Fakultät findet in ihrer kirchlichen Rechtsform und in ihrer Organisationsstruktur seinen Ausdruck, insbesondere in der Person und den Kompetenzen des Großen Vizekanzlers sowie in ihrer Bedeutung für Leben und Mission der Kirche von Tarnów. Das Ziel der Fakultät besteht in einer allseitigen und systematischen Erforschung der Göttlichen Offenbarung in den unterschiedlichen Epochen und Ausprägungen des Lebens der Kirche und des Menschen"10.

Der Titel des Periodikums "The Person and the Challenges: The Journal of Theology, Education, Canon Law and Social Studies Inspired by Pope John Paul II" lehnt sich an den Titel des Buches "Osoba i czyn"l1 (Person und Tat) von Kardinal Karol Wojtyła an, dem späteren Papst Johannes Paul II. Damit wird daran erinnert, dass nur die Person „tätig” ist, wirkt, handelt, sittliche Akte vollzieht und Geschichte erschafft. Die Person realisiert sich in ihrem Handeln, drückt sich darin aus, tritt auf und integriert. Erst durch ihr Handeln erfüllt sich die Person in ihrem Wesen und in ihrer Existenz. Nach der Vorstellung der Redakteure von "The Person and the Challenges: The Journal of Theology, Education, Canon Law and Social Studies Inspired by Pope John Paul II" werden die in dem Periodikum aufgegriffenen Inhalte die Person selbst oder im weiteren Sinn die Herausforderungen betreffen, die vor der Person stehen, die im einundzwanzigsten Jahrhundert lebt. Des weiteren geht es ebenso um Bedrohungen der Würde der Person, die aufgrund unterschiedlicher Einschränkungen nicht Person sein kann. Die Notwendigkeit, die neuartigen politischen, gesellschaftlichen und kulturellen Herausforderungen anzunehmen sowie nach einem entsprechenden christlichen Zeugnis unter qualitativ neuen Bedingungen $\mathrm{zu}$ suchen, ist daher nicht ausschließlich eine Erkenntnis der heutigen Generation. Einige Herausforderungen werden jedoch derzeit immer mehr zu einer Bedrohung. Daher besteht für all diejenigen, die zu Beginn des dritten Jahrtausends den aktuellen Herausforderungen solidarisch begegnen wollen, die gemeinschaftliche Aufgabe darin, den großen Wert all dessen zu betonen, was dem Suchen der Person Sinn verleiht. Darüber hinaus soll die Sorge um die Entwicklung der Person, um ihre ganzheitliche und integrale Erziehung und Bildung, um eine Gesellschaft der Liebe - die Zivilisation der Liebe - großen Raum einnehmen.

\footnotetext{
${ }^{9}$ Statut der Päpstlichen Universität Johannes Paul II. in Krakau, Nr. 1-2.

${ }^{10}$ Statut der Päpstlichen Universität Johannes Paul II. in Krakau, Nr. 143-144.

${ }^{11}$ Vgl. K. Wojtyła, Osoba i czyn, Kraków 2000.
} 
Die Anerkennung der Gedanken, der Reflexionen und der Lehre Johannes Pauls II. sowie der Bedarf nach einer Neuentdeckung seiner Hinweise im Kontext der Herausforderungen des einundzwanzigsten Jahrhunderts führten zur Entstehung dieser Zeitschrift. Dazu kommt das Bestreben nach wissenschaftlichem Austausch und nach unablässiger Weiterentwicklung der Wissenschaft in Einklang mit den Grundsätzen der Kirchlichen Lehre und den Anhaltspunkten für Erziehung und Bildung, die von der Europäischen Kommission in ihrem Strategiepapier „Europe $2020 " 12$ festgehalten sind.

Das internationale Periodikum "The Person and the Challenges: The Journal of Theology, Education, Canon Law and Social Studies Inspired by Pope John Paul II", eine rezensierte wissenschaftliche Zeitschrift, ist mit dem Ziel entstanden, internationale Forschungen aus den Bereichen Theologie, Bildung, Kanonisches Recht und Sozialwissenschaften zu verbreiten, die von der Person und der Lehre Papst Johannes Pauls II. inspiriert sind, indem die Diskussion um Fragen aus den oben angeführten und weiteren verwandten Gebieten angestoßen wird. In "The Person and the Challenges" sind wissenschaftlich beachtliche, bisher unveröffentlichte Arbeiten abgedruckt: Artikel, wie auch Berichte von bedeutenden Konferenzen und Buchrezensionen. Die Texte (in englischer, deutscher, spanischer, italienischer und französischer Sprache) sind als Papierversion und in digitaler Form (als PDF) auf der Internetseite der Zeitschrift publiziert. Jeder Artikel, der in einer der fünf angeführten Sprache geschrieben ist, enthält auch folgendes in englischer Sprache: Überschrift, Schlüsselwörter und eine kurze Zusammenfassung. Das Periodikum wird ab 2011, dem Jahr der Seligsprechung des Heiligen Vaters Johannes Paul II. an der Theologischen Fakultät, Sektion Tarnów, der Päpstlichen Universität Johannes Paul II. in Krakau herausgegeben.

Die Redaktion und den wissenschaftliche Beirat der internationalen Wissenschaftszeitschrift „The Person and the Challenges” bilden Forscher, die an Universitäten auf der ganzen Welt arbeiten. Entwurf und Initiative für die Entstehung des Periodikums sind Prof. Dr. habil. Józef Stala und Prof. UKSW Dr. habil. Elżbieta Osewska zu verdanken. Daher ist es verständlich, dass in der Redaktion und dem wissenschaftlichen Beirat in der Mehrzahl Professoren vertreten sind, mit denen die beiden Initiatoren bereits früher individuelle Kontakte geknüpft und die wissenschaftliche Zusammenarbeit aufgenommen haben, oder die sie durch den Austausch im „Erasmus” - Programm kennen gelernt haben. Der Redaktion des Periodikums gehören an: Prof. Dr. habil. Józef Stala (The Pontifical University of John Paul II in Cracow, Poland); Dr. Robert Kantor (The

${ }^{12}$ Vgl. http://ec.europa.eu/education/lifelong-learning-policy/doc28_en.htm (8 VIII 2011). 
Pontifical University of John Paul II in Cracow, Poland); Prof. UPJPII Dr. habil. Janusz Królikowski (The Pontifical University of John Paul II in Cracow, Poland); Prof. UKSW Dr. habil. Elżbieta Osewska (Cardinal Stefan Wyszynski University in Warsaw, Poland); Prof. KUL Dr. habil. Alicja Rynio (The John Paul II Catholic University of Lublin, Poland); Prof. UPJPII Dr. habil. Ireneusz Stolarczyk (The Pontifical University of John Paul II in Cracow, Poland). Dagegen sind der Einladung in den Internationalen Wissenschaftlichen Ehrenbeirat gefolgt: Prof. Dr. habil. Tadeusz Brzegowy (The Pontifical University of John Paul II in Cracow, Poland); Prof. Dr. Herman Lombaerts (Catholic University of Leuven, Belgium); Prof. Dr. habil. Jan Łach (Cardinal Stefan Wyszynski University in Warsaw, Poland); Prof. Dr. Hermann Rodriguez Osorio (Javeriana University of Bogota, Colombia); Prof. Dr. Flavio Pajer (Salesian Pontifical University - Rome, Italy); Prof. Dr. Graham Rossiter (Australian Catholic University, Australia); Prof. Dr. Peter Stilwell (Catholic University of Portugal, Portugal); Prof. Dr. Eloy Tejero (University of Navarrra, Spain); Prof. Dr. habil. Tadeusz Zasępa (The Catholic University in Ruzomberok, Slovakia). Die Einladung in den Internationalen Wissenschaftlichen Beirat des Periodikums haben des weiteren angenommen: Prof. Dr. Alejandro W. Bunge (Pontifical Catholic University of Argentina, Argentina); Prof. Dr. habil. Tadeusz Dola (The University of Opole, Poland); Prof. Dr. habil. Alojzy Drożdż (University of Silesia in Katowice, Poland); Prof. Dr. Roberto Jaramillo Escutia (Pontifical University of Mexico, Mexico); Prof. Dr. Franz Feiner (The Catholic University College for Teacher Training Graz, Austria); Dr. Adrian-Mario Gellel (University of Malta, Malta); Prof. Dr. Stanko Gerjolj (University of Ljubljana, Slovenia); Prof. UKSW Dr. habil. Waldemar Graczyk (Cardinal Stefan Wyszynski University in Warsaw, Poland); Prof. KULDr. habil. Andrzej Kiciński (The John Paul II Catholic University of Lublin, Poland); Dr. Patricia Kieran (Mary Immaculate College, Limerick, Ireland); Prof. UAM Dr. habil. Bogusław Kochaniewicz (Adam Mickiewicz University in Poznan, Poland); Prof. UPJPII Dr. habil. Janusz Królikowski (The Pontifical University of John Paul II in Cracow, Poland); Prof. UKSW Dr. habil. Jolanta M. Marszalska (Cardinal Stefan Wyszynski University in Warsaw, Poland); Prof. Dr. habil. Janusz Mastalski (The Pontifical University of John Paul II in Cracow, Poland); Prof. Dr. habil. Kazimierz Misiaszek (Cardinal Stefan Wyszynski University in Warsaw, Poland); Prof. UKSW Dr. habil. Elżbieta Osewska (Cardinal Stefan Wyszynski University in Warsaw, Poland); Prof. Dr. Erika Prijatelj (University of Ljubljana, Slovenia); Prof. Dr. Augusto Sarmiento (University of Navarra, Spain); Prof. Dr. habil. Józef Stala (The Pontifical University of John Paul II in Cracow, Poland); Prof. UPJPII Dr. habil. Ireneusz Stolarczyk (The Pontifical University of John 
Paul II in Cracow, Poland); Prof. UPJPII Dr. habil. Władysław Zuziak (The Pontifical University of John Paul II in Cracow, Poland).

Die Redaktion reichte die entsprechenden Dokumente beim Bezirksgericht in Tarnów, Abteilung I Zivilkammer, ein und am 30. Mai 2011 wurde das Periodikum „The Person and the Challenges" im Register für Zeitungen und Zeitschriften unter der Nummer $195^{13}$ eingeschrieben. Als Periodikum, das regelmäßig halbjährlich erscheint, erhielt „The Person and the Challenges” auch eine eigene Nummer ISSN der Nationalbibliothek.

Für die Autoren der Texte wurden unter anderem die folgenden Hinweise vorbereitet:

- Die Texte sollen im Word-Format gespeichert und als Anhang einer E-Mail oder auf einer CD übersandt werden.

- Die Artikel sollen eindeutig und klar abgefasst sein und in ihrer endgültigen Fassung eingereicht werden, darüber hinaus sollte jemand Korrektur gelesen haben, der die jeweilige Sprache fließend beherrscht (evtl. ein "Native Speaker").

- Die Herausgeber behalten sich das Recht vor, die eingereichten Texte innerhalb von drei Monaten anzunehmen oder abzulehnen. Die Texte werden von Experten des jeweiligen Fachbereichs rezensiert.

- Die Texte sollen durch Teilüberschriften in arabischer Nummerierung gegliedert sein. Die ebenfalls in arabischen Ziffern angeführten Fußnoten sind am Seitenende und nicht am Schluss des Artikels zu platzieren.

- Die in den Fußnoten angeführten Bücher und Artikel sollen folgendermaßen angegeben werden:

Bücher:

- J. Stala, Familienkatechese in Polen um die Jahrhundertwende. Probleme und Herausforderungen, Tarnów 2008, Biblos.

- E. Osewska, J. Stala (Hrsg.), Religious Education / Catechesis in the Family. A European Perspective, Warszawa 2010, UKSW.

Artikel:

- F. Pajer, L'educare e il credere tra vecchie e nuove utopie, "The Person and the Challenges" 1 (2011) 1, S. 89-98.

- P. Kieran, Family and Faith-Formation: Some Perspectives from the Irish Context, in: E. Osewska, J. Stala (Hrsg.), Religious Education / Catechesis in the Family. A European Perspective, Warszawa 2010, UKSW, S. 195-211

- http://www.wt.diecezja.tarnow.pl (6 IV 2011).

${ }^{13}$ Beschluss des Bezirksgerichts in Tarnów, Abteilung I Zivilkammer, vom 30. Mai 2011, Archiv "The Person and the Challenges". 
- Die Herausgeber behalten sich das Recht vor, Korrekturen vorzunehmen, um methodologische Standards einzuhalten.

- Der Autor erhält jeweils eine Ausgabe der Zeitschrift, in der sein Text veröffentlicht ist.

In seiner Predigt anlässlich des Requiems für Johannes Paul II. unterstrich Kardinal J. Ratzinger die große Rolle und die Vielfalt der Person und des Lehrwerks Johannes Pauls II., aber auch die Einschränkungen als Folge seiner Erkrankung und seines Leidens. „Für uns alle bleibt es unvergesslich, wie der Heilige Vater, vom Leiden gezeichnet, am letzten Ostersonntag seines Lebens noch einmal am Fenster des Apostolischen Palastes erschienen ist und zum letzten Mal den Segen »Urbi et orbi« erteilt hat. Wir können sicher sein, dass unser geliebter Papst jetzt am Fenster des Hauses des Vaters steht, uns sieht und uns segnet. Ja, segne uns, Heiliger Vater. Wir vertrauen deine liebe Seele der Mutter Gottes, deiner Mutter, an, die dich jeden Tag geführt hat und dich jetzt in die ewige Herrlichkeit ihres Sohnes, Jesus Christus unseres Herrn, führen wird"14.

Es ist zu hoffen, dass die neue internationale wissenschaftliche Zeitschrift „The Person and the Challenges: The Journal of Theology, Education, Canon Law and Social Studies Inspired by Pope John Paul II" eine Initiative sein wird, die den Blick auf die Welt von heute, auf den Menschen und die ständig neu erwachsenden Herausforderungen aus der Perspektive des Evangeliums begünstigen kann. Das Periodikum wird ab 2011, dem Jahr der Seligsprechung Johannes Pauls II. an der Universität herausgegeben, die den Namen Johannes Pauls II. trägt, damit wird gleichzeitig suggeriert und garantiert, dass sie eine Plattform für den Austausch von Gedanken, Einschätzungen und Ausarbeitungen darstellt, die den Menschen und die Herausforderungen der Gegenwart gerade im Licht oder auch in Bezug auf die Lehre Johannes Pauls II. betreffen.

${ }^{14}$ Predigt von Kard. Joseph Ratzinger anlässlich des Requiems für Johannes Paul II., Vatikan, 8. April 2005, http://www.vatican.va/gpII/documents/homily-card-ratzinger_20050408_ge.html (19.08.2011). 